\title{
Alleviation of Dyspnea Sensation by Phototherapy in Healthy Adults
}

\author{
Hideaki Izukura, Masashi Kanezaki, and Satoru Ebihara
}

\begin{abstract}
BACKGROUND: Because both dyspnea and pain have common features from a clinical and physiologic point of view, we hypothesized that stellate ganglion irradiation by using a linear polarized near-infrared ray device, which is often used for pain management, might be applicable for dyspnea relief. To evaluate the use of stellate ganglion irradiation as a novel noninvasive treatment for dyspnea, we investigated the influence of stellate ganglion irradiation on dyspnea. METHODS: Perceptions of dyspnea were examined with or without stellate ganglion irradiation in 28 healthy adults. The sensation of breathing difficulty was induced by a two-way non-rebreathing valves with linear inspiratory resistance $(R)$ of $0,10,20$, and $30 \mathrm{~cm} \mathrm{H}_{2} \mathrm{O} / \mathrm{L} / \mathrm{s}$. Dyspnea was evaluated with the modified Borg scale to determine subjects' discomfort level. Stellate ganglion irradiation was performed by intermittent irradiation near the bilateral stellate ganglion by using a linear polarized near-infrared ray device. RESULTS: There were significant changes from baseline at $\mathrm{R}=10 \mathrm{~cm} \mathrm{H} \mathrm{H}_{2} \mathrm{O} / \mathrm{L} / \mathrm{s}(P=.007), \mathrm{R}=20 \mathrm{~cm} \mathrm{H}_{2} \mathrm{O} / \mathrm{L} / \mathrm{s}(P=.005)$, and $\mathrm{R}=30 \mathrm{~cm} \mathrm{H} \mathrm{H}_{2} \mathrm{O} / \mathrm{L} / \mathrm{s}(P=.009)$. For each resistive load, the mean dyspnea sensation score was lower with stellate ganglion irradiation compared with sham irradiation, with significant differences $\left(P=.003\right.$ at $\mathrm{R}=0 \mathrm{~cm} \mathrm{H}_{2} \mathrm{O} / \mathrm{L} / \mathrm{s} ; P<.001$ at $\left.\mathrm{R}=10,20,30 \mathrm{~cm} \mathrm{H}_{2} \mathrm{O} / \mathrm{L} / \mathrm{s}\right)$. There was a significantly lower slope of the dyspnea response for the linear regression of the loads and Borg scores in the stellate ganglion irradiation versus sham treatment $(P=.003)$. CONCLUSIONS: Stellate ganglion irradiation significantly alleviated dyspnea induced by an external inspiratory load in healthy adults. Stellate ganglion irradiation might be an option to treat dyspnea in some cases. Further studies in individuals with diverse types of dyspnea and clinical settings are warranted. Key words: dyspnea; rehabilitation; control of breathing; phototherapy; stellate ganglion. [Respir Care 2019;64(9):1082-1087. (C) 2019 Daedalus Enterprises]
\end{abstract}

\section{Introduction}

Dyspnea is one of the frequent concerns reported by patients with respiratory, heart, and neurologic diseases,

Drs Izukura and Ebihara are affiliated with the Department of Rehabilitation Medicine, School of Medicine, Faculty of Medicine, Toho University, Tokyo, Japan. Dr Ebihara is affiliated with the Department of Rehabilitation Medicine, Graduate School of Medicine, Toho University, Tokyo, Japan. Dr Kanezaki is affiliated with the Department of Physical Therapy, Faculty of Health Care Sciences, Himeji Dokkyo University, Hyogo, Japan.

This study was supported by a Grants-in-Aid for Scientific Research from the Japanese Ministry of Education, Culture, Sports, Science and Technology (grants 24300187, 24659397, 26460899, 15K12588, 15K15254, and 19H03984), Research Funding for Longevity Sciences (25-7, 28-13) from the Japanese National Center for Geriatrics and Gerontology, and The Research Promotion Grant from Toho University Graduate School of Medicine (17-04 to Dr Ebihara). and with malignant tumors. In addition, it can also be caused by both exercise and aging. A previous study found that $30 \%$ of elderly people $\geq 65$ years who were not ill, reported feeling dyspnea during their daily routine activities. ${ }^{1}$ Although pulmonary rehabilitation is recommended for chronic respiratory diseases, ${ }^{2}$ exercise therapy is often restricted due to dyspnea in these patients. ${ }^{3}$ As a result, these patients cannot perform the sufficient amounts of exercise required to achieve the goal of the rehabilitation,

\footnotetext{
The authors have disclosed no conflicts of interest.

Correspondence: Satoru Ebihara MD PhD, Department of Rehabilitation Medicine, Graduate School of Medicine, Toho University, 6-11-1, Omorinishi, Ota-ku, Tokyo 143-8541, Japan. E-mail: satoru.ebihara@med. toho-u.ac.jp.
}

DOI: $10.4187 /$ respcare.06496 
such as improving exercise tolerance and/or the healthrelated quality of their lives.

Physiotherapy, such as hyperthermia, electrical muscle stimulation therapy, and hydrotherapy, are frequently used during musculoskeletal rehabilitation for the purpose of pain relief and to ensure that patients achieve sufficient amounts of exercise training. During pulmonary rehabilitation, however, such physiotherapies have not been used for symptom relief to achieve specific rehabilitation goals.

Phototherapies, for example, linear polarized near-infrared ray irradiation, have been used by many facilities, including pain clinics. Irradiation on stellate ganglion is reported to have beneficial effects on symptom management, including pain in various clinical settings. ${ }^{4-7}$ Skin burns and corneal damage are possible complications of stellate ganglion irradiation, but they seldom occur with the correct irradiation method. As for the application to respiratory diseases, Kanashiki et $\mathrm{al}^{8}$ reported a case of a patient with asthma whose dyspnea and peak expiratory flow were improved by stellate ganglion irradiation.

One of the most important features of linear polarized near-infrared ray is that it can penetrate deep within the body and supply large amounts of energy. It has been reported that both light and thermal effects can cause suppression of nerve excitation, skeletal muscle relaxation, vasodilation, and autonomic nerve regulation, and can lead to relaxation, among other things. ${ }^{9}$ Thus, these actions may be effective against respiratory muscle tension and blood-flow disorders, and sympathetic hyperactivity, all of which are factors that can exacerbate dyspnea.

Dyspnea and pain are subjective and unpleasant sensations, and have common features from a clinical, physiologic, and psychological point of view. ${ }^{10}$ Due to this commonality, the possibility exists that stellate ganglion irradiation, which is often used for pain treatment, ${ }^{4,5}$ might also be able to be used for dyspnea. Therefore, this study examined the influence of stellate ganglion irradiation on dyspnea in healthy adults for the purpose of evaluating its use as a novel treatment for this symptom.

\section{Methods}

\section{Subjects}

This study recruited 28 healthy non-smoking volunteers (20 men, 8 women; mean \pm SD age, $29.1 \pm 4.5$ y) via public postings. The subjects had no history of respiratory diseases, recent suggestive symptoms (within 4 weeks), respiratory tract infection, or seasonal allergies. No subjects took any regular medicine. The ethics committee of Toho University Faculty of Medicine approved this study (approval 27041), and all the subjects signed an informed consent form before the start of the study.

\section{QUICK LOOK}

\section{Current knowledge}

Both dyspnea and pain have common features from a clinical and physiologic point of view. Stellate ganglion irradiation by a linear polarized near-infrared ray device is often used for pain management, but the effect on dyspnea with inspiratory resistive load has not been verified.

\section{What this paper contributes to our knowledge}

Stellate ganglion irradiation significantly alleviated sensations of dyspnea induced by an external inspiratory load in healthy adults. Stellate ganglion irradiation is a safe and easy physiotherapy that does not require any special skills to perform. Stellate ganglion irradiation could be an option to relieve dyspnea in some cases. Further studies in individuals with diverse types of dyspnea and clinical settings are warranted.

\section{Perception of Dyspnea}

Dyspnea was induced by an external breathing circuit loaded with inspiratory resistance, with a modified Borg scale used to assess symptoms of breathlessness in the subjects. ${ }^{11}$ The sensation of breathing difficulty was created by a two-way non-rebreathing valves (Hans Rudolph, Kansas) with linear inspiratory resistance $(\mathrm{R})$ of 0, 10, 20 and $30 \mathrm{~cm} \mathrm{H}_{2} \mathrm{O} / \mathrm{L} / \mathrm{s}$. During each of the sessions, the respiratory resistance was progressively increased at 2-min intervals. The subjects were instructed not to control either their ventilation or breathing patterns during the test. After breathing at each load for $1 \mathrm{~min}$, the subjects were asked to rate the magnitude of "kokyu kon-nan" based on the modified Borg scale. The term "kokyu kon-nan" is an exact Japanese translation of dyspnea (kokyu means breathing or respiration, and kon-nan means difficulty or discomfort). ${ }^{12}$

The subjects were asked to sense the effort or work they expended with their ventilatory muscles during inspiration. The categories for this scale ranged from 0 (felt none) to 10 (maximum imaginable sensation). Before the measurement, the subjects were instructed to evaluate the difficulty of breathing during the test while avoiding any assessments of non-respiratory sensations, such as headache or irritation of the pharynx. ${ }^{12} \mathrm{We}$ also commented that, in contrast to air hunger, the sensation that they were feeling during breathing with a resistive load might not necessarily be uncomfortable. Because it has been reported that there is a linear relationship between the amount of load and the Borg scores, ${ }^{13,14}$ we, in addition, estimated 
the linear regression slope as the "dyspnea slope" by using a least squares fitting when the estimated Borg scores were plotted against the corresponding amounts of resistive loads.

\section{Linear Polarized Near-Infrared Ray Irradiation}

Stellate ganglion irradiation was performed by using the linear polarized near-infrared irradiation device Super Lizer PX (Tokyo Iken, Tokyo, Japan). The maximum output was $10 \mathrm{~W}$ at wavelengths of $0.6-1.6 \mu \mathrm{m}$. Conditions used during the administration of stellate ganglion irradiation in the subjects included the following: a light-emission head with a diameter of $7 \mathrm{~mm}$, an output of $80 \%$, irradiation cycle ratio of 1:2 (2 s of irradiation, followed by a 4-s pause), and an irradiation duration of $7 \mathrm{~min}$. The sternocleidomastoid muscle on the irradiated side was then gently retracted, with the probe of the device placed firmly and perpendicularly against the skin in the jugular groove at a site that was $2.5 \mathrm{~cm}$ above the costoclavicular joint. Stellate ganglion irradiation was performed on both sides. Sham irradiation was performed by using the probe of the device in the same way but with the output set to zero.

\section{Experimental Protocol}

The subjects were evaluated in the laboratory on 2 different days within 1 week. For logistical reasons, it was not possible to schedule the same time intervals for all the subjects. At $\sim 5: 00$ PM on the day of the experiment, the subjects were evaluated for perceptions of dyspnea after receiving either the sham or the stellate ganglion irradiation treatment. A random numbers table was used to select the order of the sham and stellate ganglion irradiation for each subject. Spirometry was performed on the first study day in accordance with American Thoracic Society guidelines. ${ }^{15}$ On each experimental day, we measured the perception of dyspnea.

\section{Data Analysis}

Data are expressed as the median and interquartile range except when specified otherwise. Comparisons of the Borg scores for dyspnea as a function of the inspiratory resistive loads that were imposed for 1 min with sham or stellate ganglion irradiation treatments were performed by using Wilcoxon signed-rank test with Bonferroni correction. The Wilcoxon signed-rank test was used to compare the dyspnea slopes between the sham and stellate ganglion irradiation treatments. We considered $P<.05$ to be statistically significant.

\section{Results}

None of the 28 subjects who completed the experiments had any adverse effects or uncomfortable feelings. All the
Table 1. Characteristics of the Subjects

\begin{tabular}{|c|c|}
\hline Characteristic & Results \\
\hline Age, y & $29.1 \pm 4.5$ \\
\hline Height, cm & $168.5 \pm 7.6$ \\
\hline Weight, kg & $61.9 \pm 10.1$ \\
\hline $\mathrm{FEV}_{1}, \mathrm{~L}$ & $3.73 \pm 0.57$ \\
\hline $\mathrm{FEV}_{1}, \%$ predicted & $97.6 \pm 13.2$ \\
\hline FVC, L & $4.25 \pm 0.65$ \\
\hline FVC, \% predicted & $107.2 \pm 14.6$ \\
\hline $\mathrm{FEV}_{1} / \mathrm{FVC}, \%$ & $86.8 \pm 3.87$ \\
\hline $\begin{array}{l}N=28 . \\
\text { Data are mean } \pm \text { SD. }\end{array}$ & \\
\hline
\end{tabular}

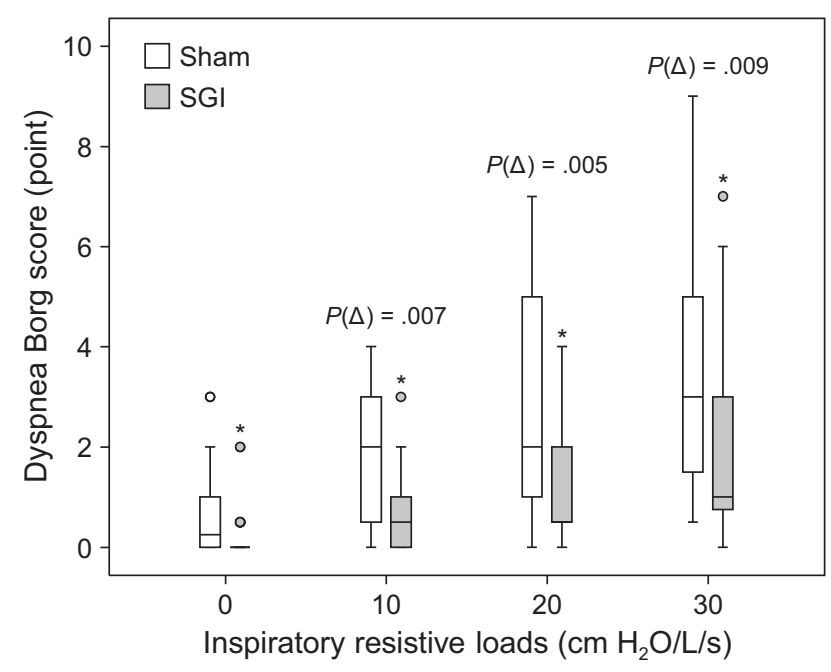

Fig. 1. Comparisons of the perception of dyspnea between sham and SGl treatments. Dyspnea sensations are shown as a function of inspiratory resistive loads imposed externally. Boxes represent interquartile ranges, with the medians shown as a horizontal lines. Bars denote maximum and minimum values. ${ }^{*} P<.001$ versus sham by using Wilcoxon signed-rank test. $P(\Delta)=$ Comparison of changes from baseline by Wilcoxon signed-rank test (adjusted for Bonferroni correction); SGI = stellate ganglion irradiation.

subjects were fit and had normal lung function. The characteristics of the subjects are summarized in Table 1. The Borg scores for dyspnea at each level of the inspiratory resistive loads that was imposed for $1 \mathrm{~min}$ are shown in Figure 1. For each resistive load, the mean dyspnea sensation was lower in the stellate ganglion irradiation compared with the sham, with significant differences $(P=.003$ at $\mathrm{R}=0 \mathrm{~cm} \mathrm{H}_{2} \mathrm{O} / \mathrm{L} / \mathrm{s} ; P<.001$ at $\mathrm{R}=10,20,30 \mathrm{~cm}$ $\left.\mathrm{H}_{2} \mathrm{O} / \mathrm{L} / \mathrm{s}\right)$. We compared changes from baseline by using the Wilcoxon signed-rank test at $\mathrm{R}=10,20,30 \mathrm{~cm}$ $\mathrm{H}_{2} \mathrm{O} / \mathrm{L} / \mathrm{s}$. There was a significant change from baseline at $\mathrm{R}=10 \mathrm{~cm} \mathrm{H}_{2} \mathrm{O} / \mathrm{L} / \mathrm{s}(P=.007), \mathrm{R}=20 \mathrm{~cm} \mathrm{H}_{2} \mathrm{O} / \mathrm{L} / \mathrm{s}$ $(P=.005)$, and $\mathrm{R}=30 \mathrm{~cm} \mathrm{H}_{2} \mathrm{O} / \mathrm{L} / \mathrm{s}(P=.009)$. The mean $\pm \mathrm{SD}$ slopes of the dyspnea response calculated by the linear regression analysis of the loads and Borg scores 


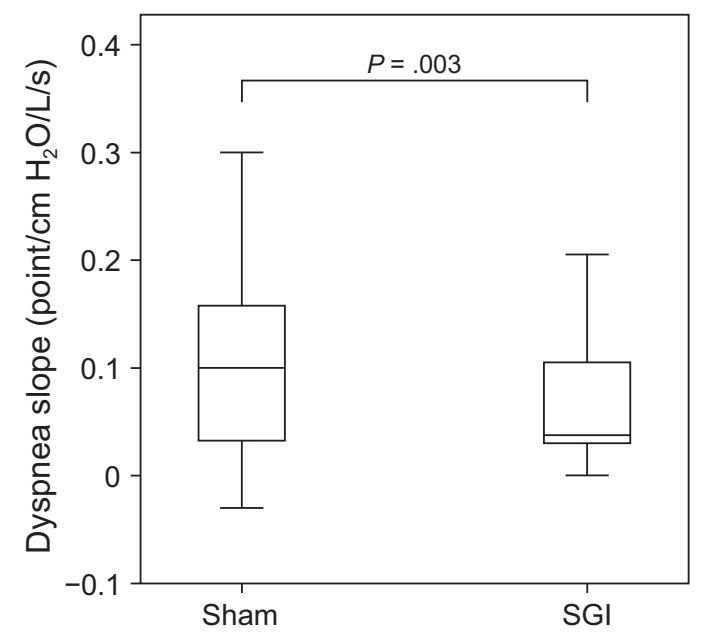

Fig. 2. Comparisons of dyspnea slope between the sham and SGI treatments. Dyspnea slope calculated by the linear regression when the dyspnea Borg scores were plotted as a function of the resistive loads. Each box represents the interquartile range, with the median value shown as a horizontal line in the box, and bars represent the maximum and minimum values. $P$ values were calculated by a Wilcoxon signed-rank test. SGI = stellate ganglion irradiation.

were lower in the stellate ganglion irradiation $(0.07 \pm 0.06)$ versus the sham treatment $(0.11 \pm 0.09)$ (Fig. 2). Significant differences were found between the slopes for the sham and stellate ganglion irradiation treatments $(P=.003)$.

\section{Discussion}

Our current study examined the effect of stellate ganglion irradiation on dyspnea in healthy adults. Analysis of the results showed that stellate ganglion irradiation significantly reduced dyspnea in healthy volunteers. Dyspnea has been defined as a subjective experience of breathing discomfort that consists of qualitatively distinct sensations that vary in intensity. ${ }^{16}$ Dyspnea and pain are both uncomfortable sensations and exhibit similarities in the clinical, physiologic, and psychological characteristics, which indicated that there may be common central pathways between both. ${ }^{10}$ Although the effect of stellate ganglion irradiation on dyspnea, to our knowledge, has not been previously reported, many studies have examined the effect of stellate ganglion irradiation on pain and investigated its potential mechanism.

Nakabeppu et $\mathrm{al}^{17}$ and Gushiken et al et al ${ }^{18}$ reported that stellate ganglion irradiation caused an increase in the thalamic blood flow that was previously reported to be decreased in subjects with chronic pain, which thereby resulted in stimulation of the descending pain suppression system and the alleviation of pain. Pain and dyspnea are relayed through a thalamic pathway, and both sensations are processed through a projection of the thalamocortical area to a particular cortical area. ${ }^{19}$ Thus, similar to that seen for pain, changes in thalamic blood flow by stellate ganglion irradiation could also play a role in the suppression of dyspnea.

Furthermore, the emotion recognition system is also connected to the sensory pathway by information from the sensory receptor via the thalamus. When dyspnea occurs, to recognize a mismatch or dissociation between the motor command and afferent information, the limbic system needs to be involved. ${ }^{20-23}$ Due to recent advances in brain imaging technology, studies have been able to investigate whether there might be a common neural circuit between dyspnea and pain. These investigations revealed that the gyrus cingli, insula, amygdala, and medial thalamus were activated, not only with dyspnea but also when pain occurred. ${ }^{24}$ However, neural connections between the stellate ganglion and the insula cortex, amygdala, and hypothalamus have been reported in the study that used trans-synaptic tracing. ${ }^{25}$ Lipov et al ${ }^{26}$ reported that stellate ganglion block was effective for treating sleep disorders of menopausal women, with inhibition of the sympathetic nerve able to suppress the hypothalamus. It has been further suggested that the relaxing effect of stellate ganglion irradiation ${ }^{9}$ could be involved in reducing dyspnea.

Liao et $\mathrm{al}^{27}$ examined the effects of stellate ganglion irradiation on pain and heart rate variability and showed that stellate ganglion irradiation improved pain and autonomic dysfunction. Although the investigators were not able to clarify the underlying mechanism, analysis of their results indicated that a coordinating sympathetic overflow and imbalance might be involved. It can, depending on the quality of the dyspnea that is present, be classified based on a sensation of work/effort, a sensation of air hunger, or a sensation of chest tightness. ${ }^{16,19}$ The dyspnea sensation used in our current study was caused by an external resistive load, which has previously been described as a sensation of work/effort. ${ }^{28}$ Similar to pain, a sensation of work/effort has been reported to be associated with a stimulation of the C-fiber in respiratory muscle and/or lung. ${ }^{29}$ Thus, stabilization of the pulmonary vagus nerve by stellate ganglion irradiation might be able to potentially reduce the dyspnea.

There are a few pharmacologic approaches available to relieve dyspnea. In pulmonary rehabilitation, it was reported that exercise endurance was enhanced by alleviating dyspnea with bronchodilators. ${ }^{30}$ In addition, the beneficial effects of opioids on dyspnea in patients with terminal cancer have also been reported. ${ }^{31}$ For patients who are seriously ill with COPD, guidelines recommend that opioids be used to relieve dyspnea. ${ }^{2}$ However, these approaches are disease specific and cannot be easily implemented due to serious adverse effects.

Nonpharmacologic approaches that have been used to help relieve dyspnea include the stimulation of the upper respiratory tract by cold air, ${ }^{32}$ nasal inhalation of men- 
thol, ${ }^{33}$ and vibrator stimulation to the chest wall accessory muscles of ventilation. ${ }^{34}$ Another study reported on the effectiveness of using whole-body thermal stimulation to improve dyspnea associated with COPD by increasing blood flow. ${ }^{35}$ Because it has also been demonstrated that stellate ganglion irradiation can significantly increase peripheral blood flow, ${ }^{36}$ it is possible that changes in ventilatory muscle blood flow could have helped relieve the dyspnea.

Conventional invasive stellate ganglion block via the use of a local anesthetic may cause severe complications, such as intra-arterial injection, esophageal puncture, and retropharyngeal hematoma. ${ }^{4}$ In contrast, the use of stellate ganglion irradiation is advantageous because it is a noninvasive procedure that can be safely performed. In addition, stellate ganglion irradiation can be administered to patients with bleeding tendencies who are undergoing anticoagulation therapy. There are skin burns as complications of stellate ganglion irradiation, but it is a rare occurrence with the correct irradiation method. Corneal damage can also be avoided by appropriately using goggles. Therefore, stellate ganglion irradiation is a safe and convenient method that can be used to relieve dyspnea in diverse situations.

Our current study had limitations. The greatest limitation in our study is that the subjects were healthy volunteers. There might be a different quality between experimental dyspnea in healthy adults and chronic sensations of dyspnea due to disease in patients. The former is a safe and experimental environment, whereas the latter is affected by anxiety and fear related to disease. The quality of dyspnea in patients with respiratory disease varies due to diverse etiologies. Dyspnea is induced not only by mechanical stresses but also by chemical stimuli, such as hypoxia, hypercapnia, inflammation, and so forth. ${ }^{37}$ In the present study, we evaluated dyspnea in a very limited situation (ie, external inspiratory resistive load). Also, differences due to age and sex must be considered. For the effect on patients, further investigation is necessary. Second, this study only used a short observation time after a single irradiation. When treating pain, Liao et $\mathrm{al}^{27}$ performed irradiation twice a week for a period of 6 weeks. Thus, for dyspnea applications, we may need to evaluate the effect of using repeated irradiation.

Sympathetic nervous activity changes are observed in patients with dyspnea. Therefore, it might be crucial to assess the effect of sympathetic nervous activities. Future research that uses autonomic nerve function evaluation and brain imaging will need to be undertaken to clarify the mechanism by which stellate ganglion irradiation relieves dyspnea. Furthermore, if the relationship of dyspnea, pain, and the effect of stellate ganglion irradiation is clarified, then this information could be useful for elucidating the fundamental mechanism linking pain and dyspnea.

\section{Conclusions}

Stellate ganglion irradiation alleviated dyspnea induced by external inspiratory load in healthy adults without any adverse effects or discomfort. Stellate ganglion irradiation is a safe and easy physiotherapy that does not require any special skills to perform. Stellate ganglion irradiation could be an option to relieve dyspnea in some cases. Further studies in individuals with diverse types of dyspnea and patients with chronic and acute dyspnea are warranted.

\section{REFERENCES}

1. Horsley JR, Sterling IJ, Waters WE, Howell JB. Respiratory symptoms among elderly people in the New Forest area as assessed by postal questionnaire. Age Ageing 1991;20(5):325-331.

2. Global Initiative for Chronic Obstructive Lung Disease (GOLD). Global strategy for the diagnosis, management, and prevention of chronic obstructive pulmonary disease. Updated 2017. http:// goldcopd.org/. Accessed April 4, 2019.

3. Garrod R, Marshall J, Barley E, Jones PW. Predictors of success and failure in pulmonary rehabilitation. Eur Respir J 2006;27(4):788794.

4. Liao CD, Tsauo JY, Chen HC, Liou TH. Efficacy of stellate ganglion blockade applied with light irradiation: a systemic review and metaanalysis. Am J Phys Med Rehabil 2017;96(6):e97-e110.

5. Yoo C, Lee WK, Kemmotsu O. Efficacy of polarized light therapy for musculoskeletal pain. Laser Therapy 1993;5(4):153-157.

6. Otsuka H, Okubo K, Imai M, Kaseno S, Kemmotsu O. Polarized light irradiation near the stellate ganglion in a patient with Raynaud's sign. Masui 1992;41(11):1814-1817.

7. Wajima Z, Shitara T, Inoue T, Ogawa R. Linear polarized light irradiation around the stellate ganglion area increases skin temperature and blood flow. Masui 1996;45(4):433-438.

8. Kanashiki H, Kikuchi K, Konishi A, Seo N. The effect of linear polarized near infrared irradiation on pulmonary function in an asthmatic patient. Jichi Med J 1999;22:295-298.

9. Ogawa S. Super Lizer (linear polarized near extra-red light irradiator). The Japanese journal of medical instrumentation 1998;68(11): 567-572.

10. Gracely RH, Undem BJ, Banzett RB. Cough, pain, and dyspnoea: similarities and differences. Pulm Pharmacol Ther 2007;20(4):433437.

11. Kikuchi Y, Okabe S, Tamura G, Hida W, Homma M, Shirato K, Takishima T. Chemosensitivity and perception of dyspnea in patients with a history of near-fatal asthma. N Engl J Med 1994; 330(19):1329-1334.

12. Gui P, Ebihara S, Kanezaki M, Suda C, Nikkuni E, Ebihara T, et al. Gender differences in perceptions of urge to cough and dyspnea induced by citric acid in healthy never smokers. Chest 2010;138(5): 1166-1172.

13. von Leupoldt A, Dahme B. Differentiation between the sensory and affective dimension of dyspnea during resistive load breathing in normal subjects. Chest 2005;128(5):3345-3349.

14. Livermore N, Butler JE, Sharpe L, McBain RA, Gandevia SC, McKenzie DK. Panic attacks and perception of inspiratory resistive loads in chronic obstructive pulmonary disease. Am J Respir Crit Care Med 2008;178(1):7-12.

15. Standardization of spirometry, 1994 Update. American Thoracic Society Am J Respir Crit Care Med 1995;152(3):1107-1136.

16. Dyspnea. Mechanisms, assessment, and management: a consensus statement. American Thoracic Society. Am J Respir Crit Care Med 1999;159(1):321-340. 


\section{Alleviation of Dyspnea With Phototherapy}

17. Nakabeppu Y, Nakajo M, Gushiken T, Tsuchimochi S, Tani A, Kanmura Y. Decreased perfusion of the bilateral thalami in patients with chronic pain detected by Tc-99m-ECD SPECT with statistical parametric mapping. Ann Nucl Med 2001;15(5):459-463.

18. Gushiken T, Nakabeppu Y, Masuyama T, Yagi Y, Tobo K, Tsuneyoshi I, et al. The effects of linear polarized infrared ray therapy on regional cerebral blood flow. Proceeding of the Fourth Congress of the World Association for Laser Therapy; 2002 Jun 27-30; Tokyo, Japan. Bologna: Edito Grafica; 2002:135-139.

19. Nishino T. Dyspnoea: underlying mechanisms and treatment. Br J Anaesth 2011;106(4):463-474.

20. Evans KC, Banzett RB, Adams L, McKay L, Frackowiak RS, Corfield DR. BOLD fMRI identifies limbic, paralimbic, and cerebellar activation during air hunger. J Neurophysiol 2002;88(3):1500-1511.

21. Schwartzstein RM, Manning HL, Weiss JW, Weinberger SE. Dyspnea: a sensory experience. Lung 1990;168(4):185-199.

22. Banzett RB, Mulnier HE, Murphy K, Rosen SD, Wise RJ, Adams L. Breathlessness in humans activates insular cortex. Neuroreport 2000; 11(10):2117-2120.

23. Peiffer C, Poline JB, Thivard L, Aubier M, Samson Y. Neural substrates for the perception of acutely induced dyspnea. Am J Respir Crit Care Med 2001;163(4):951-957.

24. von Leupoldt A, Sommer T, Kegat S, Baumann HJ, Klose H, Dahme B, Büchel C. Dyspnea and pain share emotion-related brain network. Neuroimage 2009;48(1):200-206.

25. Westerhaus MJ, Loewy AD. Central representation of the sympathetic nervous system in the central cortex. Brain Res 2001;903(12):117-127.

26. Lipov EG, Joshi JR, Sanders S, Wilcox K, Lipov S, Xie H, et al. Effects of stellate-ganglion block on hot flushes and night awakenings in survivors of breast cancer: a pilot study. Lancet Oncol 2008; 9(6):523-532.

27. Liao CD, Rau CL, Liou TH, Tsauo JY, Lin LF. Effects of linearly polarized near-infrared irradiation near the stellate ganglion region on pain and heart rate variability in patients with neuropathic pain. Pain Med 2017;18(3):488-503.
28. Lansing RW, Im BS, Thwing JI, Legedza AT, Banzett RB. The perception of respiratory work and effort can be independent of the perception of air hunger. Am J Respir Crit Care Med 2000;162(5): 1690-1696.

29. Morélot-Panzini C, Demoule A, Straus C, Zelter M, Derenne JP, Willer JC, Similowski T. Dyspnea as a noxious sensation: inspiratory threshold loading may trigger diffuse noxious inhibitory controls in humans. J Neurophysiol 2007;97(2):1396-1404.

30. Casaburi R, Kukafka D, Cooper CB, Witek TJ Jr, Kesten S. Improvement in exercise tolerance with the combination of tiotropium and pulmonary rehabilitation in patients with COPD. Chest 2005; 127(3):809-817.

31. Bruera E, MacEachern T, Ripamonti C, Hanson J. Subcutaneous morphine for dyspnea in cancer patients. Ann Intern Med 1993; 119(9):906-907.

32. Spence DP, Graham DR, Ahmed J, Rees K, Pearson MG, Calverley PM. Does cold air affect exercise capacity and dyspnea in stable chronic obstructive pulmonary disease? Chest 1993;103(3):693-696.

33. Nishino T, Tagaito Y, Sakurai Y. Nasal inhalation of 1-menthol reduces respiratory discomfort associated with loaded breathing. Am J Respir Crit Care Med 1997;156(1):309-313.

34. Homma I, Obata T, Sibuya M, Uchida M. Gate mechanism in breathlessness caused by chest wall vibration in humans. J Appl Physiol 1984;56(1):8-11.

35. Kikuchi H, Shiozawa N, Takata S, Ashida K, Mitsunobu F. Effect of repeated Waon therapy on exercise tolerance and pulmonary function in patients with chronic obstructive pulmonary disease: a pilot controlled clinical trial. Int J Chron Obstruct Pulmon Dis 2014;9: 9-15.

36. Liao CD, Tsauo JY, Liou TH, Chen HC, Rau CL. Efficacy of noninvasive stellate ganglion blockade performed using physical agent modalities in patients with sympathetic hyperactivity-associated disorders: a systematic review and meta-analysis. PLoS One 2016; 11(12): e0167476.

37. Mahler DA, O'Donnell DE. Recent advances in dyspnea. Chest 2015; 147(1):232-241. 